\title{
IMPROVEMENT IN THE APPROACHES TO THE TREATMENT OF EMIGRANTS AND RE-EMIGRANTS WITH DEPRESSIVE DISORDERS
}

\author{
O. P. Venger, Y. I. Mysula, L. V. Shkrobot \\ I. HORBACHEVSKY TERNOPIL STATE MEDICAL UNIVERSITY, TERNOPIL, UKRAINE
}

Background. Social and clinical relevance of emigration and re-emigration is determined by a significant amount of Ukrainians that emigrate and re-emigrate. Clinical features of depressive disorders have not been studied; as well as risk factors and antiriscs of various forms of depression have not been established yet. Development of comprehensive approaches to the treatment and rehabilitation of these patients is an effective way of resolving of this problem and is of significant scientific, practical, medical and social importance.

Objective. The development of measures for treatment of depressive disorders in emigrants and re-emigrants is based on investigation and analysis of clinical-psychopathological phenomenology of depression.

Methods. We examined 196 emigrants, 191 re-emigrants and 198 not emmigrants with final clinical diagnosis of depressive disorder according to ICD-10 criteria. Examination was conducted by means of depression rate according to M. Hamilton, the Montgomery-Asberg and Symptom Checklist-90-Revised and the scale of life quality by WHO QOL-26. Statistical analysis of the results was performed using nonparametric methods: Mann-Whitney test, Wilcoxon criterion of signs.

Results. It was found that the maximum content of organic acids is accumulated in grass Hyssopus officinalis L. (Lamiaceae), and the minimal is in the leaves of Chrysánthemum xhortorum L. variety Apro (Asteraceae). The dominance of aliphatic acids was determined by means of gas chromatography. Benzoic is predominant among aromatic acids.

Conclusions. The suggested therapy schemes proved higher effectiveness compared with the traditional one, and also allowed to improve the life quality of emigrants and re-emigrants.

KEY WORDS: depressive disorders; emigrants; re-emigrants; clinical-psychopathological features.

\section{Introduction}

Emigration and re-emigration is one of the most important and actual public and social matter. The number of Ukrainian emmigrants is estimated up to 6.5-7 million people, what is about $15 \%$ of the population, and tends to increase [7]. Re-emigration (coming back of emigrants) is independent medical and social problem; its amounts are comparable with migration. Migration is a traumatic factor, provoking manifestation and exacerbation of endogenous mental disorders [1]. In the structure of personality changes in emigrants, emotional instability, anxiety, social introversion, behavioral conformity, suspicion, poor integration of personality traits as emotivity and frustration, low level of frustrational tolerance are detected. Among mental disorders associated with emi-

Corresponding author: Olena Venger, Department of Neurology, Psychiatry, Narcology and Medical Psychology, I. Horbachevsky Ternopil State Medical University, 1 Maidan Voli, Ternopil, Ukraine, 46001

Phone number: +380352435774

E-mail: venger_ol@tdmu.edu.ua gration, psyhodisadaptative states, posttraumatic stress disorder, neurasthenia, prolonged depressive reaction and moderate depressive episode are the most common.

However, despite the significance of the problem of emigration and re-emigration important issues related to the course of depressive disorders in emigrants and re-emigrants are have not been studied, treatmentrehabilitation programs have not been developed and implemented for emigrants and re-emigrants with depressive disorders.

The aim of the study is the development and improvement of measures for treatment of depressive disorders in emigrants and re-emigrants due to investigation and analysis of clinical-psychopathological phenomenology of depression.

\section{Methods}

We examined 196 persons who for at least one last year lived outside Ukraine and planned to return abroad soon (emigrants); and 191 persons who at least a year lived outside Ukrai- 
ne and over the last year have returned for permanent residence in Ukraine (re-emigrants). Also we included 198 persons which were permanently living in the Ukraine, and never traveled abroad for long stay (not emmigrants). All of the patients were treated in the Ternopil Regional Municipal Clinical Psychoneurological Hospital for the period from 2010-2014 years, with established final clinical diagnosis of depressive disorder according to ICD-10 criteria. Psychogenic depressive disorder (ICD-10 codes F43.21 and F43.22) was established in 69 of not emigrants, 68 of emigrants and 67 re-emigrants, endogenous (ICD-10 codes F31.3, F31.4, F32.1, F32. 2, F33.1 and F33.2) - in 65, 66 and 63 persons accordingly, organic (ICD-10 code F06.3) - in 64, 62 and 61 people accordingly. Examination was conducted by means of depression rate according to M. Hamilton [8], the MontgomeryAsberg [9] and questionnaire of psychopathological symptoms severity Symptom Checklist90-Revised [10] and the scale of life quality by WHO QOL-26. Statistical analysis of the results was performed by nonparametric methods: Mann-Whitney test, Wilcoxon a criterion of signs.

\section{Results}

The determined in our study clinical-psychopathological and patopersonal changes in emigrants and re-emigrants prove the necessity of improvement of psychiatric help for this group of patients. Today in Ukraine the absence of targeted treatment and rehabilitation programs for re-emigrants and emigrants determines the necessity of development of funda mental principles, aims and approaches to the treatment of depressive disorders in these patients.

Considering the clinical-psychopathological and patopersonological features evidenced during the examination of the emigrants and re-emigrants with depressive disorders of different genesis, we recommended the following schemes of medicational therapy and psychotherapy.

The suggested scheme of treatment and rehabilitation due to the principles of an integrated, individual and differentiated approach, ensure the staging and continuity of treatment and rehabilitation. Its objectives defined as more rapid elimination of depressive disorders (depression, anxiety-depressive, asthenic-depressive, apatho-depressive syndromes), maximal recovery and social functioning of patients, prevention of recurrence of depressive disorders, providing early socialization and social adaptation considering the features found in the emigrants and re-emigrants, engaging families and public organizations. Clinical features of depressive disorders (depressed mood, anhedonia, fatigue, anxiety, agitation, etc.), social desadaptation and disorders of microsocial interaction, abnormal behavioral patterns associated with the presence of depressive disorders, as well as factors of emigration and re-emigration were defined as therapy goals.

The suggested scheme was realized in four stages that consisted of succession and continuity of treatment and diagnostic measures. The first stage - diagnostical - included clinical psychopathological assessment of disorders that were evidenced in the patient's psychoemotional sphere, analysis of anamnesis, clinical symptoms, dynamics and prognosis of the disease, the relationship of clinical and psychosocial factors. The second stage - complex treatment - included a compound of biological therapy and psychotherapy aimed to relieve depressive disorder, normalization of emotional state, social adaptation and readaptation. Antidepressant therapy at this stage included prescription of agomelatine in a daily dose of $25 \mathrm{mg}$ for the emigrants suffering from psychogenic depressive disorders, for the patients with endogenous depressive disorders - quetiapin in a daily dose of $200 \mathrm{mg}$, for the emigrants with depressive disorders of organic genesis - fluoxetinein a daily dose of $20 \mathrm{mg}$, for the re-emigrants with psychogenic depressive disorders mirtazapine in a daily dose of $30 \mathrm{mg}$, for the re-emigrants with endogenous depressive disorders - combination of fluoxetine and risperidone in a daily dose of $20 \mathrm{mg}$ and $2 \mathrm{mg}$ accordingly, for the re-emigrants with depressive disorders of organic genesis - fluoxetine in daily dose of $20 \mathrm{mg}$. Differentiated psychotherapeutic correction for the emigrants included rational use of psychotherapy, cognitive behavioral therapy and group psychotherapy, and for the emigrants suffering from psychogenic depressive disorder - also psyhoeducational therapy. Psychotherapeutic interventions for the reemigrants consisted of rational, family, cognitive-behavioral therapy; the focus was on working with the automatic thoughts and dysfunctional beliefs. The criterion of treatment efficacy was stable (at least two weeks) normalization of emotional state, disappearance of clinical signs of depressive disorder, working out adequate emotional response to real-life circumstances, including emigration (re-emi- 
gration), disactualization of destructive and formation of constructive patterns of behavior. The third stage - rehabilitation - aimed at forming stable adequate emotional-behavioral pattern, maximum adaptation and readaptation of a patient, preventing of depressive disorder relapse, while medicamental therapy was similar to that used during the treatment with the appropriate dosage correction due to the actual condition of the patient. Differentiated psychotherapeutic work included the use of cognitive-behavioral therapy for the emigrants, and a combination of cognitive-behavioral therapy, family therapy and autogenous training for the re-emigrants. At this stage, also the measures for rehabilitation and social reintegration of the patients were also useful. The fourth stage - preventive - aimed at maintaining of normal emotional state, effective stress resistance and prevention of depressive disorder recurrence. Medical therapy included treatment of primary disease in cases of depressive disorders of organic genesis and seasonal prevention of depressions of endogenous character and psychotherapy - techniques of selfregulation, and measures for social adaptation.

Comparison analysis of effectiveness was conducted in three main directions: 1. Dynamics of mental state (recovery, significant improvement of mental state, improvement of mental state, lack of dynamics of mental state, worsening of mental state). 2. Dynamics of pathological manifestations severity according to psychometric scales. 3. Dynamics of life quality rate during the treatment. Reassessment performed 6 months after the beginning of the treatment.

To evaluate the effictiveness of the suggested treatment, regimens were divided into 6 major groups due to the emigrants and reemigrants suffering from psychogenic, endogenous and organic depressive disorders, receiving the suggested treatment and 6 similar in all clinical and social characteristics comparison groups. Comparison of the results was conducted in pairs of each clinical group due to the clinical variant and genesis of depressive disorder. Comparing analyzes of effectiveness was conducted in three main directions: dynamics of mental state, dynamics of pathological manifestations severity according to psychometric rate and dynamics of life quality during treatment.

Assessment of mental state dynamics as a result of the conducted therapy proved significantly higher efficiency of the offered scheme of treatment compared to the traditional one (Fig. 1).

In the main group higher quality of treatment was achieved: in the group of the emigrants suffering from psychogenic depressive disorders in $81.2 \%$ of the patients clinical recovery, in $9.4 \%$ - a significant improvement, in $6.3 \%$ - improvement, no dynamics was detected in one patient $(3.1 \%)$, cases of reverse dynamics of depressive disorders under the influence of treatment were not found, while in the control group of patients clinical recovery was achieved in $42.4 \%$ of cases, significant improvement - in $33.3 \%$, improvement - in $15.2 \%$, no changes - in $6.1 \%$, and in one case (3.0\%) inverse dynamics $(p<0.05)$ was evidenced. In the emigrants with endogenous depressive disorder, who received the suggested scheme of treatment, clinical recovery was achieved in $59.3 \%$ of cases, significant improvement - in $21.9 \%$, improvement - in $12.5 \%$, lack of dynamics - in $6.3 \%$, in the group of patients receiving traditional therapy clinical recovery was achieved in $24.2 \%$, a significant improvement and improvement - in $27.3 \%$, no dynamics was detected in $18.2 \%$, worsening of state - in one case $(3.0 \%)(p<0.05)$. In the group of emmigrants with depressive disorders of organic genesis, clinical recovery was achieved in $36.6 \%$, a signi-

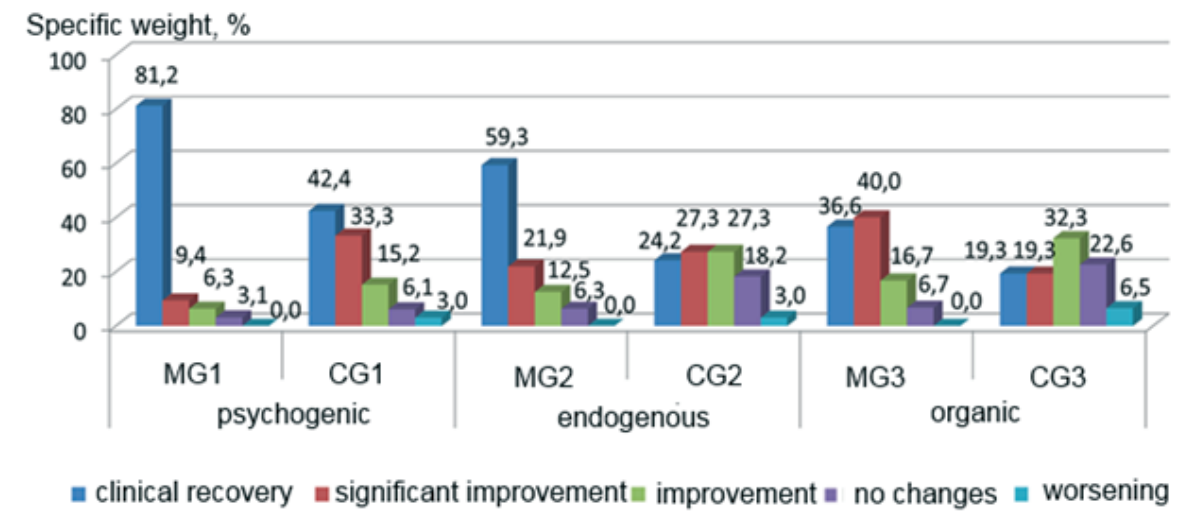

Fig. 1. Comparison of the results of traditional and suggested treatment schemes for the emmigrants, patients with depressive disorders. 
ficant improvement - in 40.0\%, improvement in $16.7 \%$, lack of dynamics - in $6.7 \%$, in the comparison group clinical recovery was found in $19.3 \%$, a significant improvement - in $19.3 \%$, improvement - in 32.3\%, no explicit dynamics - in $22.6 \%$, worsening of state - in $6.5 \%(p<0.05)$.

In the re-emigrants with depressive disorders higher effectiveness of the suggested scheme was also proved (Fig. 2).

In the re-emigrants suffering from psychogenic depressive disorder treated by the suggested scheme, clinical recovery was achieved in $71.0 \%$ of cases, significant improvement - in $16.1 \%$, improvement - in $9.7 \%$, the lack of dynamics occurred in one case (3.2\%), the inverse dynamics was not detected, and there-emigrants with psychogenic depressive disorders who received conventional therapy achieved clinical recovery in $32.2 \%$, a significant improvement - in $35.5 \%$, improvement - in $22.6 \%$, no dynamics was detected in $6.5 \%$, inverse dynamics - in $3.2 \%$ of the patients $(p<0.05)$. In the re-emigrants with endogenous depressive disorders, treated by traditional therapy, clinical recovery was achieved in $51.5 \%$ of cases, significant improvement - in $22.6 \%$, improvement in $19.4 \%$, no dynamics was detected in $6.5 \%$ of the patients. After the treatment by the suggested clinical therapy, recovery and significant improvement occurred in $19.3 \%$, improvement - in $42.0 \%$, lack of dynamics - in $12.9 \%$, inverse dynamics - in $6.5 \%$ of the patients $(p<0.05)$. In the re-emigrants with depressive disorders of organic genesis, the suggested scheme has lead to clinical recovery in $33.3 \%$, a significant improvement - in $36.7 \%$, improvement - in $20.0 \%$, no dynamics was detected in $10.0 \%$, and in the comparison group of clinical recovery occurred in $13.3 \%$, a significant improvement $20.0 \%$, improvement - in $40.0 \%$, lack of dynamics - in $16.7 \%$, state worsening - in $10.0 \%$ of the patients $(p<0.05)$.

\section{Discussion}

Due to the the intended aim and objectives of our study the analisis of the received results made it possible to justify the treatment and rehabilitation programs for the emigrant and re-emigrant patients with depressive disorders. Considering the features of depressive disorders in these groups of patients and lack of information on the developed approaches to treatment and rehabilitation, we suggested new therapeutic schemes. Therefore, we invite you to the discussion of scientific research that proved positive results.

Analysis of quantitative indices by scale of depression according to M. Hamilton also confirmed significantly higher efficiency of the suggested scheme for both the emigrant and re-emigrant patients with psychogenic, endogenous and organic depressive disorders.

So, the suggested scheme of therapy for the emigrants suffering from psychogenic depressive disorder was significantly more effective against depressed mood, guilt, suicidal intentions, early, middle and late insomnia, working capacity and activity, retardation, agitation, mental anxiety, somatic symptoms, genital symptoms and hypochondria. The suggested scheme also promoted to significant decrease of the overall rate of depression and indicators of adynamic, agitated depression, depression with fear and undifferentiated depression.

The suggested scheme of treatment enabled achievement of significantly improved indexes of main subrates of M. Hamilton depression scale in the emigrants with endogenous depressive disorders. Significant differences were detected by comparison of the indexes after reasonable treatment of depressed mood, guilt, early, middle and late insomnia, working capacity and activity, retardation, agitation, mental anxiety, somatic symptoms and hypochondria, as well as general indicators of de-

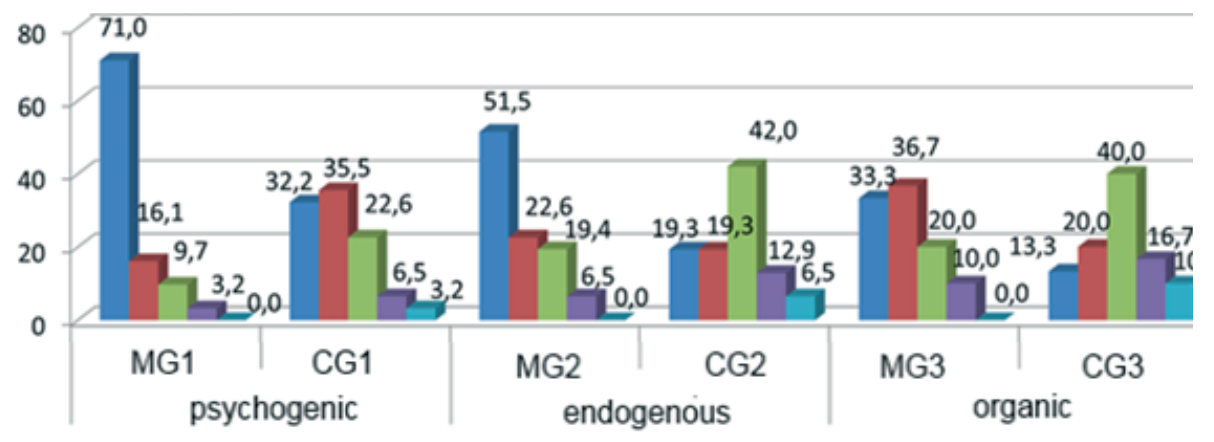

" clinical recovery = significant improvement $=$ improvement $\mid$ no changes $\mid$ worsen

Fig. 2. Comparison of the results of traditional and suggested treatment schemes for the re-emigrants with depressive disorders. 
pression, indexes of adynamic, agitated depression, depression with fear and undifferentiated depression.

For the emigrants suffering from depressive disorders of organic genesis, the suggested scheme of treatment was significantly more effective against depressed mood, guilt, suicidal intentions, early, middle and late insomnia, working capacity and activity, retardation, agitation, mental anxiety, somatic symptoms, genital symptoms and hypochondria, as well as general indicators of depression, indexes of adynamic, agitated depression, depression with fear and undifferentiated depression.

The suggested scheme of treatment also allowed decreasing anxiety in the re-emigrants and the emigrants with depressive disorders of different genesis.

For the emigrants suffering from psychogenic depressive disorders, the suggested scheme was significantly more effective against anxious mood, tension, anxiety, insomnia, depressed mood, muscle somatic symptoms, somatic sensory symptoms, cardiovascular symptoms, respiratory symptoms, hastrointestional symptoms, autonomic symptoms, as well as general severity of psychic and somatic anxiety.

For the emigrants with endogenous depressive disorder, the suggested scheme was significantly more effective against anxious mood, tension, insomnia, depressed mood, muscle somatic symptoms, somatic sensory symptoms, cardiovascular symptoms, respiratory symptoms, vegetative symptoms, as well as general severity of psychic and somatic anxiety.

For the emigrants suffering from depressive disorders of organic nature, the suggested scheme was significantly more effective against anxious mood, tension, anxiety, insomnia, depressed mood, muscle somatic symptoms, cardiovascular symptoms, respiratory symptoms, vegetative symptoms, as well as general severity of psychic and somatic anxiety.

In the re-emigrants the positive influence of the suggested scheme was evidenced. Thus, for the re-emigrants suffering from psychogenic depressive disorder, the suggested scheme was more effective against anxious mood, tension, anxiety, insomnia, cognitive disorders, depressed mood, muscle somatic symptoms, somatic sensory symptoms, cardiovascular symptoms, respiratory symptoms, gastrointestinal symptoms, vegetative symptoms, as well as general severity of psychic and somatic anxiety.

The suggested scheme of treatment for the re-emigrants with endogenous depressive disorder, was more effective against anxious mood, tension, anxiety, insomnia, cognitive disorders, depressed mood, muscle somatic symptoms, somatic sensory symptoms, cardiovascular symptoms, respiratory symptoms, gastrointestinal symptoms, vegetative symptoms, as well as the general severity of psychic and somatic anxiety.

For the re-emigrants with depressive disorders of organic genesis, the suffested scheme significantly improved the rate of anxious mood, anxiety, insomnia, muscle somatic symptoms, somatic sensory symptoms, cardiovascular symptoms, respiratory symptoms, gastrointestinal symptoms and severity of psychic and somatic anxiety.

The influence of the suggested therapy on the severity of psychopathological symptoms was also more effective compared to the traditional one.

For the emigrants suffering from psychogenic depressive disorders, the suggested scheme proved significantly better results in the rate of somatization, obsessive-compulsive disorders, interpersonal sensitivity, depression, anxiety, hostility, phobic anxiety and general indicator (index GSI), the index of symptoms detecting (PSI) and index of distress severity (PDSI).

For the emigrants suffering from depressive disorders of organic genesis, the suggested scheme was proved effectiveness in the rate of of somatization, obsessive-compulsive disorders, interpersonal sensitivity, depression, anxiety, hostility, phobic anxiety and general indicator (index GSI), the index of symptoms detecting (PSI) and index of distress severity (PDSI).

In the patients with depressive disorders of different genesis significantly better rates of psychopathological symptoms severity influenced by the suggested scheme of therapy were achieved.

Thus, the re-emigrants suffering from psychogenic depressive disorders, treated by the suggested scheme achieved more pronounced decrease in the rates of somatization, obsessive-compulsive disorders, interpersonal sensitivity, depression, anxiety, hostility, phobic anxiety and general indicator (index GSI), the index of symptoms detecting (PSI) and index of distress severity (PDSI).

For the re-emigrants with endogenous depressive disorders, the suggested therapy scheme promoted to more effective decrease in the rates of somatization, obsessive-compul- 
sive disorders, interpersonal sensitivity, depression, anxiety, hostility, phobic anxiety and general indicator (index GSI), the index of symptoms detecting (PSI) and index of distress severity (PDSI).

For the re-emigrants with depressive disorders of organic genesis, the suggested treatment scheme was more effective for decrease in rates of somatization, obsessive-compulsive disorders, interpersonal sensitivity, anxiety, hostility, phobic anxiety and general indicator (index GSI), the index of symptoms detecting (PSI) and index of distress severity (PDSI).

Significant improvement in quality of life during the treatment is an important factor that positively assesses the perspectives of implementation of the suggested treatment regimens.

Thus, in the group of emigrants suffering from psychogenic depressive disorders, the suggested scheme promoted to achievement of significant improvement of physical health and psychological QOL.

For the emigrants with endogenous depressive disorders, the use of the suggested scheme of therapy promoted to improvement of QOL in physical health, psychological QOL and environment.

For the emigrants suffering from depressive disorders of organic genesis, the suggested scheme was more effective in improvement of QOL in physical health and psychological QOL.

The improvement of QOL during the treatment by the suggested scheme was also achieved in the re-emigrants. Thus, in the re-emigrants with psychogenic depressive disorders, significantly higher QOL of physical health and

\section{References}

1. De Wit M, Tuinebreijer W, Dekker J, et al. Depressive and anxiety disorders in different ethnic groups M. Social Psychiatry and Psychiatric Epidemiology. 2008;43(11):905-912.

2. Hamilton M. Rating scale for depression by Max Hamilton. Journal of Neurology, Neurosurgery, Psychiatry. 1960;23:56-62.

3. Hynie M, Crooks VA, Barragan J. Immigrant and refugee social networks: determinants and consequences of social support among women newcomers to Canada. Canadian Journal of Nursing Research. 2011;43:26-46.

4. Gavin BE, Kelly BD, Lane A, et al. Mental health of emigrants. Irish Medical Journal. 2001;94:229-230.

5. Guruge S, Thomson MS, George U, Chaze F. Social support, social conflict, and immigrant wo- psychological QOL were defined, in the reemigrants with endogenous depressive disorders - of QOL in physical health, psychological QOL and environment, in the re-emigrants with depressive disorders of organic origins - of QOL in physical health, psychological QOL and environment.

Thus, the comparative analysis of the suggested treatment schemes for the emigrants and re-emigrants suffering from psychogenic, endogenous and organic depressive disorder proved significantly higher effectiveness comparing with traditional one.

\section{Conclusions}

The analysis of the influence of the suggeste therapy schemes on the severity of psychopathological symptoms also proved higher effectiveness compared with the traditional one; it was evidenced by significant decrease in the rate of somatization, obsessive-compulsive disorders, interpersonal sensitivity, depression, anxiety, hostility, phobic anxiety, as well as general index, the index of symptoms detection and the index of distress severity.

The suggested therapy scheme of also promoted to improvement of life quality of the emigrants and re-emigrants with depressive disorders, significant increase in the rates of their physical health, psychological quality of life and environment compared to the comparison group.

The results allow recommending the developed treatment scheme for implementation in the complex treatment of emigrants and reemigrants suffering from psychogenic, endogenous and organic depressive disorders. men's mental health in a Canadian context: A scoping review. Journal of Psychiatric and Mental Health Nursing. 2015;22:655-667.

6. Lauber C, Lay B, Rossler W. Patterns of Psychiatric Inpatient Care in Migrants: Results from Switzerland. Swiss Medical Weekly. 2006;135:50-56.

7. Migration in Ukraine: facts and numbers. International organization of migration. Kyiv: Representation IOM in Ukraine; 2011;7.

8. Montgomery SA, Asberg M. A new depression scale designed to be sensitive to change. British Journal of Psychiatry. 1979;134:382-389.

9. Selten JP, Veen N, Feller W, et al. Incidence of psychotic disorders in immigrant groups to the Netherlands. British Journal of Psychiatry. 2001; 178:367-372.

Received: 2016-12-29 